\title{
BIOLOGIA DA POLINIZAÇÃO EM LECYTHIDACEAE
}

Scott Alan Mori (1)

\begin{abstract}
RESUMO - A família Lecythidaceae apresenta grande diversidade em matas higrófilas localizadas abaixo de $1000 \mathrm{~m}$ de altitude. Poucas espécies ocorrem em cerrados, várzeas, e em matas acima de $1000 \mathrm{~m}$. O Brasil, onde ocorrem $54 \%$ das espécies de Lecythidaceae do Novo Mundo, tem mais espécies desta família que qualquer outro país no mundo, sendo especialmente rico èm espécies com flores zigomorfas. A evoluçāo floral ocorreu principalmente no androceu que pode ser do tipo actinomorfo ou do tipo zigomorfo. Os polinizadores principais são abelhas sendo a única exceçâo a polinização por morcegos em Lecythis poiteaui. As abelhas da tribo Euglossini parecem ser muito importantes na evoluçẫo de Lecythidaceae com flores zigomorfas. As flores de Lecythidaceae oferecem três recompensas aos seus polinizadores: pólen nẫo diferenciado, pólen diferenciado, e néctar. Ainda há muito para pesquisar sobre biologia da reprodução em Lecythidaceae. Estudos sobre a composição química do pólen, a quantidade e qualidade do néctar, e o sistema de compatibilidade sāo especialmente importantes.
\end{abstract}

\begin{abstract}
The family Lecythidaceae is most diverse in lowland, moist forests below $1000 \mathrm{~m}$ altitude. Only a few species are found in savannas, periodically inundated habitats, and forests above $1000 \mathrm{~m}$. Brasil, where $54 \%$ of the New World species occurs, has more species of Lecythidaceae than any other country. It is especially diverse in species with zygomorphic flowers. Floral evolution in Lecythidaceae has taken place mostly in the androecium where actinomorphic and zygomorphic types have developed. The principal pollinators are bees with the only documented exception being the bat pollinated Lecythis poiteaui. Euglossine bees appear to be particularly important in the evolution of zygomorphic flowered Lecythidaceae. The flowers of Lecythidaceae, depending on the species, offer three kinds of rewards to pollinators - undifferentiated pollen, differentiated pollen, and nectar. There is still much to be learned about reproductive biology in Lecythidaceae. Studies of pollen chemistry, nectar quantity and quality, and self incompatibility systems are especially needed.

Key-words: Lecythidaceae, floral biology.
\end{abstract}

Introdução

A familia Lecythidaceae, sensu latu, abrange quatro subfamilias das quais três são, principalmente, do Velho Mundo (Planchonioideae, Foetidioideae e Napoleonaeoideae) e uma que é inteiramente do Novo Mundo (Lecythidoideae). A única exceção é Asteranthos brasiliensis Desfontaines, uma Napoleonaeoideae da várzea do alto rio Negro (Mori \& Prance 1981). Neste trabalho, abordo somente as Lecythidoideae, portanto quando me refiro a Lecythidaceae ou Lecitidáceas, estou indicando esta subfamilia.

As Lecitidáceas incluem desde árvores pequenas até muito grandes que crescem principalmente em matas higrófilas abaixo de 1000 metros de altitude. A maioria das espécies encontra-se na terra firme mas algumas preferem a várzea (e.g., Allantoma lineata (Mart. ex Berg) Miers, Couratari oligantha A. C. Smith, C.tenuicarpa A. C. Smith, Eschweilera ovalifolia (DC) Niedenzu, E. parvifolia Mart. ex DC., e E. tenuifolia (Berg) Miers. Somente 14 espécies, todas de Eschweilera, das 200 espécies do Novo Mundo encontram-se acima de 1000 metros de altitude. Poucas espécies crescem em cerrados. Eschweilera nana (Berg) Miers tem ampla distribuição no cerrado do Planalto do Brasil. Cariniana rubra Gardner ex Miers, Lecythis miersiana Mori, e L. schomburgkii Berg ocorrem em cerradc mas com distribuição geográfica bem menores que Eschweilera nana. 
O Brasil possui mais espécies de Lecythidaceae do que qualquer outro país no Novo Mundo. Das 200 espécies da regiảo neotropical, 108 (54\%) ocorrem no Brasil. Ainda mais impressionante é a diversidade de espécies brasileiras com flores zigomorfas. Das 137 espécies com este tipo de flor, 81 (59\%) encontram-se apenas ou parcialmente no Brasil. Quanto às espécies com flores actinomorfas, somente 27 das 63 espécies (43\%) são nativas no Brasil. As espécies de Cariniana, com flores ligeiramente zigomorfas, foram consideradas como actinomorfas para esta análise; portanto, o número de espécies com flores completamente actinomorfas no Brasil é ainda menor. Grias, um gênero de seis espécies com flores actinomorfas, é o único sem representantes brasileiros. Ao contrário, Allantoma, Asteranthos, Bertholletia, Cariniana, Corythophora, Couratari, Couroupita, e Lecythis estāo representados por mais de dois terços das suas espécies neste país.

\section{Fenologia}

Em áreas com estações secas definidas, as espécies de Lecythidaceae florescem principalmente na estação seca e frutificam no começo da estação chuvosa (Mori \& Prance 1987a, 1987b). Por exemplo, no centro da Guiana Francesa, onde ocorre uma estação seca de agosto a novembro, 26 das 27 espécies de Lecythidaceae florescem nesta ápoca do ano. Na Guiana Francesa, a grande maioria das espécies dispersam suas sementes no começo da estação chuvosa (Mori \& Prance 1987b).

Onde não há estação seca definida, outros fatores podem servir como estímulo à floração. Por exemplo, no sul da Bahia, onde não ocorrem meses do ano com menos chuva, a sapucaia (Lecythis pisonis Cambess.) floresce ano após ano ao final do inverno até o final da primavera. Este fato sugere que a sapucaia inicia a floração em resposta ao aumento no comprimento do dia e/ou temperatura, fatores que ocorrem na primavera (Mori et al. 1980).

\section{Polinização}

O androceu das Lecitidáceas é caracterizado por muitos estames soldados na base, formando um anel, o qual pode ser actinomorfo (simétrico) em Allantoma, Gustavia, Grias, ou zigomorfo (assimétrico) em Bertholletia, Corythophora, Couroupita, Couratari, Eschweilera, e Lecythis. $\mathrm{O}$ androceu em Cariniana é intermediário, sendo levemente zigomorfo. Limito minha discussão ao androceu porque é nesta parte da flor onde tem ocorrido a evolução em resposta à seleção para determinados polinizadores. Desenhos de todos os tipos de androceu encontram-se em Mori e Prance (1981).

As flores actinomorfas sempre são abertas e de fácil acesso a qualquer polinizador enquanto que as flores zigomorfas podem ser abertas ou fechadas. Por exemplo, no abricó de macaco (Couroupita guianensis Aublet), uma espécie com flor zigomorfa, existe um espaço entre o capuz e o anel estaminal do androceu. Na castanha-do-Pará (Bertholletia excelsa Humb. \& Bonpl.), ao contrário, o capuz se posiciona bem junto ao anel estaminal e as pétalas são apertadas ao androceu. No primeiro caso, qualquer inseto pode entrar na flor, enquanto que, no segundo caso, somente insetos relativamente grandes com força adequada podem penetrar na flor.

Todos os estudos feitos indicam que abelhas são os principais polinizadores de Lecythidaceae (Mori et al. 1978, Mori \& Boeke 1987, Moritz 1984, Müller et al. 1980, Ormond et al. 1981, Prance 1976). A única exeçâo conhecida até agora é a polinização por morcegos em Lecythis poiteaui Berg (Mori et al. 1978). Um grupo de abelhas, a tribo Euglossini, parece ser muito importante na polinização de Lécythidaceae com flores zigomorfas. Estas abelhas são visitantes freqüentes das flores zigomorfas mas pouco vistas em Lecythidaceae com flores actinomorfas. As distribuições geográficas de Euglossini e de Lecythidaceae com flores zigomorfas quase coincidem, e os dois grupos são limitados 
ao Novo Mundo. A única divergência nas suas distribuiçōes é a presença de uma espécie de Euglossini na Jamaica onde não há nenhuma espécie de Lecythidaceae com flores zigomorfas (Mori \& Boeke 1987).

As flores de Lecythidaceae oferecem três tipos de recompensa aos polinizadores: pólen não diferenciado, pólen diferenciado, e néctar. Todas as espécies com flores actinomorfas oferecem somente pólen não diferenciado. Nestas espécies, o pólen que as abeIhas coletam é o mesmo que efetua a fecundação. Nas espécies zigomorfas, algumas produzem pólen diferenciado como recompensa às abelhas. As abelhas retiram da flor um tipo de pólen, que não germina, para alimentar suas larvas, enquanto um outro tipo de pólen, o que germina, fica na cabeça e nas costas da abelha na posiçāo certa para polinizar a próxima flor visitada. Exemplos deste sistema de polinizaçāo encontram-se em Couroupita guianensis e Lecythis pisonis (Mori \& Orchard 1979, Ormond et al. 1981). O pólen não diferenciado é coletado somente pelas fêmeas das abelhas. Finalmente, algumas espécies de Lecythidaceae produzem um néctar que é colhido por abelhas de ambos os sexos. Todas as espécies de Bertholletia, Couratari, Eschweilera e algúmas espécies de Lecythis possuem somente néctar como recompensa aos polinizadores (Mori \& Boeke 1987).

\section{Futuros Estudos}

Ainda há muito para pesquisar sobre a biologia da reprodução em Lecythidaceae. Estudos sobre compatibilidade genética em Lecythidaceae são escassos. Os poucos estudos que existem sugerem que as espécies são alógamas (Mori \& Kallunki 1976, Moritz 1984). Estudos detalhados do sistema de compatibilidade só existem para Bertholletia excelsa (Moritz 1984, Müller 1980) e Couroupita guianensis (Ormond et al. 1981), portanto estudos de outras espécies são ainda necessários.

Por outro lado, mais observações no campo são necessárias para confirmar ou modificar as observações já feitas. Por exemplo, seria interessante testar a hipótese sobre a preferência de abelhas Euglossini por flores zigomorfas. Também, estudos de espécies simpátricas de Lecythidaceae devem ser feitas para melhor entender como tantas espécies de Lecythidaceae podem ocorrer numa mesma mata.

Necessitamos dados sobre a composição química do pólen diferenciado e sobre a quantidade e composição do néctar. Será que o pólen diferenciado tem substâncias necessárias para o desenvolvimento das abelhas que o coletam?

Devido ao grande tamanho da maioria das espécies de Lecythidaceae, estudos sobre polinização são difíceis de efetuar. Por outro lado, no cerrado do Brasil Central ocorre Eschweilera nana, uma espécie de baixo porte, que pode ser estudada facilmente para obtermos respostas para estas e outras perguntas da biologia da polinização em Lecythidaceae.

\section{Agradecimentos}

Agradeço à Nanuza Luiza de Menezes pelo convite para participar no XXXVIII Congresso Nacional de Botânica e a Marlies Sazima pela ajuda editorial. Muitas das idéias expressas neste trabalho foram desenvolvidas juntamente com meu colega Ghillean T. Prance, cuja colaboração constante em nossos estudos sobre Lecythidaceae eu agradeço. 


\section{Referências Bibliográficas}

MORI, S. A. \& BOEKE, J. D. 1987. Chapter XII. Pollination. In: S. A. Mori \& collaborators, The Lecythidaceae of a lowland neotropical forest: La Fumée Mountain, French Guiana. Mem. New York Bot. Gard. 44: 137-155.

MORI, S. A. \& KALLUNKI, J. A. 1976. Phenology and floral biology of Gustavia superba (Lecythidaceae) in central Panama. Biotropica 8: 184-192.

MORI, S. A. \& ORCHARD, J. E. 1979. Fenologia, biologia floral e evidência sobre dimorfismo do pólen de Lecythis pisonis Cambess. (Lecythidaceae). Anais do XXXI Congresso Nacional de Botânica, Sociedade Botânica do Brasil p. 109-116.

MORI, S. A. \& PRANCE, G. T. 1981. Relações entre a classificação genérica de Lecythidaceae do Novo Mundo e seus polinizadores e dispersadores. Revta brasil. Bot. 4: 31-37.

MORI, S. A. \& PRANCE, G. T. 1987a. Species diversity, phenology, plant-animal interactions, and their correlation with climate, as illustrated by the Brazil nut family (Lecythidaceae). In: R. E. Dickinson (ed.), The Geophysiology of Amazonia. John Wiley \& sons, New York, p. 69-89.

MORI, S. A. \& PRANCE G. T. 1987b. Chapter XI. Phenology. In: S. A. Mori \& collaborators, The Lecythidaceae of a lowland neotropical forest: La Fumée Mountain, French Guiana. Mem. New York Bot. Gard. 44: 124-136.

MORI, S. A., PRANCE, G. T.\& BOLTEN, A. B. 1978. Additional notes on the floral biology of neotropical Lecythidaceae. Brittonia 30: 113-130.

MORI, S. A., MATTOS SILVA, L. A. \& SANTOS, T. S. dos. 1980. Observaçōes sobre a fenologia e biologia floral de Lecythis pisonis Cambess. (Lecythidaceae). Revista Theobroma (Brasil) $1 O(3)$ : 103-11.

MORITZ, A. 1984. Estudos biológicos da castanha-do-Brasil (Bertholletia excelsa H.B.K.). EMBRAPA, Centro de Pesquisa Agropecuário do Trópico Umido. Documentos 29: 1-82.

MÜLLER, C. H., RODRIGUES, 1. A., MUULLER, A.\& MÜLLER, N. R. M. . 1980. Castanha-do-Brasil. Resultados de pesquisa. EMBRAPA, Centro de Pesquisa Agropecuário do Trópico Umido. Miscelânea 2: $1-25$.

ORMOND, W. T., PINHEIRO, M. C. B. \& CORTELLA DE CASELLS, A. R. 1981. A contribution to the floral biology and reproductive system of Couroupita guianensis Aubl. (Lecythidaceae). Ann. Missouri Bot. Gard. 68 : 514-523.

PRANCE, G. T. 1986. The pollination and androphore structure of some Amazonian Lecythidaceae. Biotropica 8: $235-241$. 